\title{
Reflets
}

Revue d'intervention sociale et communautaire

\section{Analyse intersectionnelle de l'oppression linguistique de mères racisées victimes de violence conjugale en contexte montréalais}

\author{
Dominique Damant, Jeanne Bernard, Marianne Chbat et Catherine Flynn
}

Volume 21, numéro 1, printemps 2015

Violences faites aux femmes et contextes minoritaires

URI : https://id.erudit.org/iderudit/1032551ar

DOI : https://doi.org/10.7202/1032551ar

Aller au sommaire du numéro

Éditeur(s)

Reflets, Revue d'intervention sociale et communautaire

ISSN

1203-4576 (imprimé)

1712-8498 (numérique)

Découvrir la revue

Citer cet article

Damant, D., Bernard, J., Chbat, M. \& Flynn, C. (2015). Analyse intersectionnelle de l'oppression linguistique de mères racisées victimes de violence conjugale en contexte montréalais. Reflets, 21(1), 137-160.

https://doi.org/10.7202/1032551ar
Résumé de l'article

Le présent article rend compte des diverses formes d'oppression linguistique que certaines mères racisées, victimes de violence conjugale, ont rencontrées sur le plan structurel et interpersonnel en contexte montréalais. Ces résultats sont tirés d'une recherche plus large portant sur les expériences de maternité de femmes autochtones et de femmes racisées en contexte de violence conjugale.
Tous droits réservés @ Reflets, Revue d’intervention sociale et communautaire, 2015
Ce document est protegé par la loi sur le droit d'auteur. L'utilisation des services d'Érudit (y compris la reproduction) est assujettie à sa politique d'utilisation que vous pouvez consulter en ligne. 


\section{Analyse intersectionnelle de l'oppression linguistique de mères racisées victimes de violence conjugale en contexte montréalais}

Dominique Damant, Ph. D.

Professeure titulaire, École de service social, Université de Montréal Jeanne Bernard

Étudiante à la maitrise, École de service social, Université de Montréal Marianne Chbat

Étudiante au doctorat en sciences humaines appliquées, Université de Montréal

Catherine Flynn, Ph. D.

École de service social, Université de Montréal

Résumé

Le présent article rend compte des diverses formes d'oppression linguistique que certaines mères racisées, victimes de violence conjugale, ont rencontrées sur le plan structurel et interpersonnel en contexte montréalais. Ces résultats sont tirés d'une recherche plus large portant sur les expériences de maternité de femmes autochtones et de femmes racisées en contexte de violence conjugale.

Mots clés : oppression linguistique, intersectionnalité, violence, femmes racisées, mères 
This article reports on various linguistic forms of oppression that some racialized mothers victim of domestic violence encountered on a structural and interpersonal level in the context of Montreal. These results are drawn from a larger research which focused on the the experiences of Aboriginal and racialized mothers living in the context of domestic violence in various provinces of Canada.

Key words: linguistic oppression, intersectionality, violence, racialized women, mothers

«I feel like here they want me to speak French and if you don't speak French then shame on you kind of thing $[\ldots]$ even if a person can speak English, a lot of time they will pretend they can't speak English, because they feel you are in my province, why won't you speak my language. Well they don't take into consideration I just got here. Give me time to learn [...]" I don't feel we take the time to find out.You know, to go beyond the iceberg to see what people really are all about.We just assume.You're here in Montreal, or Quebec. You should be speaking French. I got here a month ago. And I'm willing to learn French and I think I learn a lot considering that I just got here. But I need money to go to the French school. Give me the money and I will go. And even if I don't need to pay for the school, I need to take the bus to get there. » (Yellow Flower)

\section{Introduction}

La violence conjugale, un mal en soi répandu, affecte les femmes racisées de façon spécifique (Damant et Lapierre, 2012). Ces dernières sont plus susceptibles d'être victimes de violence sévère (Humphreys, et collab., 2006) et de rencontrer des difficultés reliées 
"Puisqu'il

est impossible

d'homogénéiser

l'expérience des

femmes racisées, il apparaît primordial

d'analyser leur

expérience en la

mettant en lien avec

le contexte social, culturel, historique et politique dans lequel elle s'inscrit. » particulièrement au racisme, à la religion, à la structure familiale, à la langue ou au statut légal d'immigration (Dobash et Dobash, 2000). Puisqu'il est impossible d'homogénéiser l'expérience des femmes racisées, il apparait primordial d'analyser leur expérience en la mettant en lien avec le contexte social, culturel, historique et politique dans lequel elle s'inscrit. Le féminisme intersectionnel nous semble le cadre théorique le plus pertinent pour en jauger la complexité. Malgré la richesse des écrits théoriques sur le féminisme intersectionnel, peu de recherches empiriques mobilisent ce cadre théorique dans l'analyse de leurs données. Une première recherche sur l'expérience de mères victimes de violence conjugale à l'aide du féminisme intersectionnel (Damant, et collab., 2008) nous avait pourtant convaincues de sa pertinence. Cependant, la constitution de notre population (femmes blanches, francophones et en situation de pauvreté de la grande région de Québec) en limite selon nous l'utilisation. Dans le but de complexifier nos analyses, une seconde subvention (Damant, CRSH, 2009-2012) nous a permis de rencontrer des femmes confrontées à de multiples formes de marginalisation. Ainsi, nous avons rencontré des femmes racisées de la région de Montréal et des femmes des Premières Nations de l'Ouest canadien; selon les données récentes, ces femmes sont parmi les plus opprimées au Québec et au Canada. Même si leur vécu d'oppression diffère historiquement, socialement et culturellement, nous voulions relever les éléments spécifiques à leurs expériences de maternité en contexte de violence. Le présent article vise donc à analyser à la lumière du féminisme intersectionnel les résultats de cette recherche ${ }^{1}$. Les objectifs de celle-ci portaient sur l'ensemble des formes d'oppression vécues par ces mères et sur les stratégies qu'elles adoptent. Cependant, dans le présent article, nous ne tenons compte que des formes d'oppression spécifiquement reliées à la langue - quoiqu'absentes des propos des mères des Premières Nations de l'Ouest canadien majoritairement anglophone ${ }^{2}-$, car à notre étonnement, la question linguistique est apparue comme centrale dans les propos de nos répondantes montréalaises lors de la codification des données. Nous avons dû en tenir compte dans notre analyse. Après une courte introduction sur la francisation 
au Québec, nous présenterons le cadre d'analyse de la recherche, sa méthodologie et l'analyse de ses résultats.

\section{Problématique}

La question linguistique chez les personnes issues de l'immigration au Québec demeure à ce jour incontournable. Elle a fait l'objet de nombreux écrits scientifiques au cours des années 1980 et 1990 (Brédimas-Assimopoulos, 1983; Bataille, Mc Andrew et Potvin, 1998; Castonguay, 1997; Helly, 1994; Hohl, 1996; Labelle, 1989;1990; Mc Andrew et Jacquet, 1996; McAll, 1992; Molinaro et Ego, 1996; Piché, 1992; Turcotte, 1997). En fait, la francisation au Québec prend son véritable essor en 1977 avec l'adoption de la Charte de la langue française, mieux connue sous le nom de "Loi 101 ", qui succédait à la Loi sur la langue officielle de 1974 ou Loi 22 (Office québécois de la langue française, 2013). Le texte de la Loi 101 (Office québécois de la langue française, 2013; 2014) repose sur quatre assises :

- La charte impose l'usage exclusif du français dans l'affichage public et la publicité commerciale;

- La charte étend les programmes de francisation à toutes les entreprises employant cinquante personnes ou plus;

- La charte restreint l'accès à l'école anglaise aux seuls enfants dont l'un des parents a reçu son enseignement primaire en anglais au Québec;

- La charte reconnait la version française des lois comme étant la seule qui soit officielle.

Préalablement, nous assistons en 1968 à la fondation du ministère de l'Immigration du Québec (aujourd'hui ministère de l'Immigration, de la Diversité et de l'Inclusion). Son mandat porte sur l'établissement d'immigrants au Québec et sur leur contribution au développement de la province tout en permettant leur adaptation au milieu québécois et le maintien de leurs cultures d'origines. Ce projet politique se poursuit avec la mise sur pied de centres d'orientation et de formation des immigrants (COFI) qui ont pour mission d'enseigner les deux langues officielles 
"Ces initiatives

culminent en 1975

par l'inscription dans

la Charte des droits

et libertés du Québec

du droit au respect de la culture d'origine et à l'interdiction de la discrimination sur la base de la langue et de l'origine ethnique ou nationale..." aux personnes immigrantes souhaitant intégrer le marché du travail. Ces initiatives culminent en 1975 par l'inscription dans la Charte des droits et libertés du Québec du droit au respect de la culture d'origine et à l'interdiction de la discrimination sur la base de la langue et de l'origine ethnique ou nationale (Helly, 1994). C'est donc avec l'adoption de la Loi 101 que la langue française est érigée en véhicule de référents identitaires historiques, linguistiques et culturels au Québec. D'ailleurs, dès 1978, La Politique québécoise du développement culturel rendue publique dévoile que la convergence vers un projet culturel collectif se substitue à la juxtaposition des cultures. Près de 10 ans après l'adoption de la Loi 101, en 1988, les COFI sont appelés à étendre leurs services aux personnes réfugiées ou immigrantes ne s'apprêtant pas à entrer sur le marché du travail (Helly, 1994). Aujourd'hui, une diversité de cours de français est offerte à la population immigrante, allant de cours intensifs à temps complet à des cours spécialisés, en passant par des cours à temps partiel et d'autres offerts en milieu de travail, chacun d'eux étant assujetti à des critères particuliers d'admissibilité (Immigration, Diversité et Inclusion Québec, 2014). À ce sujet, Calinon (2009) souligne que la formation linguistique octroyée par le Ministère démontre un intérêt et une ouverture politiques à l'égard des immigrants. Pourtant, il ressort du degré de maîtrise de la langue que les cours de francisation dans leur forme actuelle demeurent insuffisants, ce qui nuit à l'intégration professionnelle des immigrants allophones. La formation s'en tient à la transmission de compétences linguistiques et sociolinguistiques et sa brièveté autant que son intensité ne conduisent qu'à une faible mobilité sociale. Ce constat s'avère encore plus important pour les personnes venues d'Asie. Il va sans dire qu'une partie de la population immigrante vit une insécurité linguistique. Selon Calinon (2009), le Québec aurait plus intérêt à orienter la formation vers la compréhension de la langue parlée par les Québécois et la socialisation plutôt que de s'en tenir à l'usage de la langue à des fins utilitaires et économiques. Depuis la mise en place d'un attirail de structures politiques et législatives autour de la question de l'immigration, de la langue et de la culture, les gouvernements qui se sont succédé à la tête 
"Aujourd'hui, le contexte économique de compression, la montée du néoconservatisme et l'exode du travail ouvrier sont à prendre en compte..." de la province ont mis en place une série de plans d'action visant l'intégration des nouveaux arrivants. Cela dit, pour Molinaro et Ego (1999), l'intégration linguistique ne se mesure pas de façon précise, d'autant plus dans un contexte linguistique québécois qui est double. Il n'en reste pas moins que cette intégration linguistique se vit de multiples façons chez les allophones qui parfois font preuve de résistance, mais qui cherchent surtout à surmonter leurs difficultés socioéconomiques et à dépasser leur impuissance politique.

En 1983, une étude de Brédimas-Assimopoulos se penche sur le rapport à la langue française qu'entretiennent les Grecs issus de l'immigration d'après-guerre et installés au Québec au cours des années 1950 et 1960. À cette époque, la population étudiée ne se doute pas, avant de s'y installer, que le Québec a le français comme langue majoritaire; les répondants perçoivent l'anglais comme la langue dominante en Amérique du Nord. En raison de leur appartenance religieuse, les jeunes Grecs sont dirigés vers les écoles protestantes anglophones; cela entraîne l'anglicisation d'une partie notable d'immigrants venus vivre au Québec. Le groupe sondé note par ailleurs une indifférence des francophones à leur égard. À la lumière de ses résultats, Brédimas-Assimopoulos (1983) conclut qu'on ne peut qu'étudier la question de l'intégration ethnolinguistique en observant la situation spécifique d'un groupe ethnique et les circonstances historiques rencontrées dans la société d'accueil. En revanche, l'immigration récente au Québec revêt un visage qui differe grandement de celui des années 1960 en pleine révolution tranquille. Aujourd'hui, le contexte économique de compression, la montée du néoconservatisme et l'exode du travail ouvrier sont à prendre en compte (Labelle, 1989; 1990). Dans le même ordre d'idées, Bélanger, Sabourin et Lachapelle (2011) s'attardent aux appartenances linguistiques des immigrants au Québec en examinant leur âge, leur lieu de résidence, leur origine ethnoculturelle, leur niveau d'éducation et la dynamique exogamique. Ils considèrent également que l'apprentissage d'une langue nouvelle doit sa réussite, entre autres, à l'exposition à cette langue dans le pays d'origine et aux ressources en temps et en argent dont on dispose dans le pays 
"...ce racisme de classe est similaire au sexisme de classe dans la mesure où il incorpore des notions de division sexuelle $d u$ travail." d'établissement. De son côté, McAll (1992) soutient que cette même intégration est influencée par l'âge, le sexe, la provenance ethnique, les compétences linguistiques acquises, le statut d'immigration, le niveau de scolarité et l'expérience de travail, sans compter la situation socioéconomique dans lequel se trouve le pays d'établissement. De plus, l'auteur se penche brièvement sur les trajectoires socioprofessionnelles des femmes immigrantes qui s'avèrent surreprésentées dans le secteur manufacturier du textile et dans celui des travaux domestiques. Il soutient alors que le marché de l'emploi est compartimenté selon les appartenances ethnolinguistiques francophone, anglophone et allophone. Cette compartimentation devient encore plus complexe au Québec en raison de la dualité entre l'usage du français et de l'anglais. McAll (p. 127) parle de "racialisation de la langue » où le travail manuel se trouve dévalorisé par rapport à son homologue qu'est le travail langagier (intellectuel). Pour lui, ce racisme de classe est similaire au sexisme de classe dans la mesure où il incorpore des notions de division sexuelle du travail. On ne peut donc ignorer les rapports d'inclusion et d'exclusion entre les communautés linguistiques et les groupes ayant un statut minoritaire au Québec.

On ne peut passer outre le fait que la forte immigration internationale au Québec assure la croissance démographique dans un contexte de dénatalité. Selon les données du recensement de 2006, on compte en moyenne 50000 immigrants reçus par année pour une population d'environ 8 millions d'habitants (Bélanger, Sabourin et Lachapelle, 2011). Piché (1992) remarque que l'intégration linguistique difficile des immigrants est perçue comme une menace à la "culture québécoise d'origine ". À cet effet, Mc Andrew et Jacquet (1996) rapportent que selon les acteurs du secteur public en éducation la sélection et l'intégration des immigrants sur la base linguistique sont insuffisantes; selon eux, c'est sur le plan de l'arrimage socioculturel qu'il faut miser. Toujours dans le domaine de la scolarisation, Hohl (1996) indique que le personnel enseignant et administratif des milieux scolaires s'attend à ce que les parents d'élèves partagent les mêmes normes sociales et culturelles en ce qui a trait à la participation au projet éducatif des enfants. C'est ce qui ressort d'une étude portant sur les 
"Il est rare que soient pris en compte la position précaire des parents immigrants et leur manque de ressources culturelles, linguistiques et matérielles. Par conséquent, le personnel scolaire déduit à tort que les parents allophones et analphabètes se soucient peu de la réussite scolaire de leurs enfants. » rapports entre le personnel des écoles et les parents salvadoriens et haïtiens. Il est rare que soient pris en compte la position précaire des parents immigrants et leur manque de ressources culturelles, linguistiques et matérielles. Par conséquent, le personnel scolaire déduit à tort que les parents allophones et analphabètes se soucient peu de la réussite scolaire de leurs enfants. En ce sens, la formation du personnel scolaire est requise pour saisir les multiples composantes de l'enseignement et de l'accompagnement d'élèves en contexte de pluriethnicité (Kanouté, et collab., 2008). Parallèlement, Fortin (2001) s'intéresse au point de vue des immigrants, plus spécialement de Francophones immigrant au Québec - entre autres, d'origine marocaine et algérienne selon lequel l'usage préalable du français ne garantit pas l'inclusion sociale. D'après leur expérience dans la société d'établissement québécoise, ils demeurent des "minorités audibles » aux yeux des autres francophones (p. 78) et ne s'expriment pas dans le "vrai québécois " (p. 79).

Toujours en ce qui a trait à l'éducation, depuis 1977, l'école de langue française est le lieu d'enseignement le plus fréquenté par les immigrants. En 2010, Gagné est allée à la rencontre de plusieurs « enfants de la Loi 101 » qui ont complété leur scolarité depuis quelques années. Ces personnes rapportent que le français occupe une place importante dans leur vie et la plupart disent comprendre les implications sociales et politiques de l'utilisation de cette langue au Québec. Du même coup, elles n'ont pas délaissé leur langue maternelle et restent attachées à ce bagage linguistique. Dans la même veine, en 1971, 90 \% des enfants allophones étaient inscrits à l'école anglophone. En 1994-1995, la situation est renversée : $79 \%$ d'allophones fréquentent un milieu scolaire francophone (Molinaro et Ego, 1999). En contrepartie, même si plusieurs mesures ont été prises pour assurer l'égalité des chances pour les communautés linguistiques autres que francophones que la Loi 101 est venue partiellement ébranler, au chapitre des retards et des échecs scolaires, on retrouve encore selon Bataille, Mc Andrew et Potvin (1998) une surconcentration d'enfants issus de certaines minorités linguistiques; l' « inadaptation des politiques scolaires et des contenus d'enseignement ", l'« incompréhension 
culturelle» et les «tensions interethniques » sont en cause (p.130). Il n'est donc pas rare de constater que les immigrants ont du ressentiment envers la société d'établissement et qu'ils adoptent des réflexes de protection identitaire.

Les effets de la Loi 101 ne se font pas ressentir que dans le milieu éducatif. Les retombées des politiques linguistiques sur l'évolution de l'usage du français sont importantes (Castonguay, 1997). L'intégration socioéconomique des immigrants à travers les années est plus difficile au Québec que dans le reste du Canada (Labelle, 1989). Le prisme de la langue est souvent emprunté pour étudier cette composante de l'immigration. Notamment, McAll (1992, p. 120) s'attarde à la "rentabilité des attributs linguistiques ». Il rapporte qu'en 1970, à scolarité et expérience égale, les hommes allophones avaient des revenus semblables à leurs homologues unilingues francophones. En 1980, après l'adoption de la Loi 101, les allophones se retrouvent moins bien rémunérés que les unilingues francophones, et ce, dans la moitié des cas. Dès 1986, les travailleurs unilingues francophones jouissent de salaires similaires à ceux des travailleurs unilingues anglophones. En parallèle, les allophones sont globalement désavantagés (1992). Vingt ans plus tard, Reichhold et Jacob (2012), soutiennent qu'au Québec, on parvient difficilement à intégrer les nouveaux arrivants au marché du travail. Les chiffres du recensement de 2011 indiquent que le Québec réussit moins bien que le reste

"Pour eux, l'enjeu réel de l'immigration n'est pas le nombre d'immigrants accueillis, mais bien le choix institutionnel, une erreur stratégique qui est en partie responsable d'un taux de chômage et de décrochage scolaire important. » du Canada à ce chapitre. Le plan d'action gouvernemental La diversité : une valeur ajoutée (2008-2013) n'est pas indifférent à ce constat. Il cherche à bonifier les services d'intégration sociale, professionnelle et linguistique offerts aux immigrants. Toujours selon Reichhold et Jacob (2012), les caractéristiques de l'intervention au Québec sont en partie tributaires de cet échec : on n'alloue que $10 \%$ des budgets aux initiatives communautaires et locales, et ce, au profit des institutions (ministères, conseils, offices, etc.), alors que dans le reste du Canada, c'est $70 \% \mathrm{du}$ budget qu'on leur accorde. Pour eux, l'enjeu réel de l'immigration n'est pas le nombre d'immigrants accueillis, mais bien le choix institutionnel, une erreur stratégique qui est en partie responsable d'un taux de chômage et de décrochage scolaire important. Sur 
"...le fait de ne posséder que l'anglais au Québec donne lieu à une faible mobilité sociale et professionnelle.... les répondants ayant des compétences linguistiques restreintes rapportent souffrir d'isolement, avoir un réseau social restreint, bénéficier de peu de moyens de se mélanger à la société québécoise et s'adonner à très peu d'échanges interculturels. » cette même lancée, les politiques linguistiques québécoises n'ont pas de pendants infaillibles en matière de francisation. Dans son étude, Calinon (2009) rapporte le témoignage des immigrants qui se prononcent sur leur sentiment d'intégration; la capacité de communiquer favorise ce sentiment, sans compter la contribution d'autres éléments intégrateurs. La maîtrise du français leur permet de répondre à leurs besoins primaires à leur arrivée au pays, d'intégrer le marché du travail à moyen terme et de s'intégrer socialement à long terme. D'après les résultats de l'étude, le fait de ne posséder que l'anglais au Québec donne lieu à une faible mobilité sociale et professionnelle. Cependant, le bilinguisme, lui, semble assurer une mobilité sociale et professionnelle, plus que l'unilinguisme francophone. Du moins, les répondants ayant des compétences linguistiques restreintes rapportent souffrir d'isolement, avoir un réseau social restreint, bénéficier de peu de moyens de se mélanger à la société québécoise et s'adonner à très peu d'échanges interculturels.

Tout compte fait, à la lumière de cette exploration partielle de la littérature portant sur l'usage de la langue française au Québec et l'expérience des immigrants, nous constatons que la question a souvent été abordée sous l'angle de la réussite scolaire des jeunes (Hohl, 1996; Kanouté et collab., 2008; Gagné, 2010; Mc Andrew et Jacquet, 1996; Bataille, Mc Andrew et Potvin, 1998; Reichhold et Jacob, 2012; Calinon, 2009), de l'intégration socioéconomique (McAll, 1992; Presnukhina, 2011; Reichhold et Jacob, 2012; Calinon, 2009) ou des effets des politiques sociales depuis l'adoption de la Charte de la langue française en 1977 (BrédimasAssimopoulos, 1983; Helly, 1994; Labelle, 1989; 1990; Turcotte, 1997; Molinaro et Ego, 1999; Bélanger, Sabourin, et Lachapelle, 2011; Ouellet, 2011). En contrepartie, nous constatons aux premiers abords que très peu d'études mettent en avant-scène le discours des personnes issues de l'immigration. De surcroit, nous n'avons pas repéré d'études s'attardant au point de vue des femmes immigrantes sur la question linguistique. Et même si plusieurs éléments d'analyse y ont été cités en plus de celui d'immigrantes allophones, les travaux n'ont pas présenté l'enchevêtrement de multiples éléments identitaires pour comprendre pleinement les 
systèmes d'oppression dont les personnes immigrantes et racisées font l'objet, d'où la pertinence des données que nous nous apprêtons à présenter.

\section{Cadre d'analyse}

"Cette analyse permet d'aller au-delà de la multiplicité des systèmes d'oppression en se concentrant également sur la façon dont l'interaction entre ces derniers (re) produit des inégalités sociales..."
Notre cadre d'analyse se centre essentiellement sur le féminisme intersectionnel. Comme le souligne Bilge (2009, p.70), ce paradigme de recherche renvoie à une théorie transdisciplinaire visant à appréhender la complexité des identités et inégalités sociales par une approche intégrée. Elle réfute le cloisonnement et la hiérarchisation des grands axes de différenciation sociale que sont les catégories de sexe/genre, classe, race, ethnicité, âge, handicap et orientation sexuelle.

Cette analyse permet d'aller au-delà de la multiplicité des systèmes d'oppression en se concentrant également sur la façon dont l'interaction entre ces derniers (re)produit des inégalités sociales (Bilge, 2009; Brah et Phoenix, 2004; Collins, 2000; Crenshaw, 1989). Elle permet d'appréhender la réalité sociale des acteurs ainsi que les dynamiques s'y rattachant comme étant multiples et déterminées simultanément de façon interactive par plusieurs axes d'organisation sociale (Stasiulis, 1999). L'analyse peut s'opérer simultanément sur le plan macrosociologique et microsociologique (Bilge, 2009) et va au-delà de l'incidence des catégories sociales de l'expérience. Elle appréhende ces dernières comme partie intégrante d'un cadre plus large en portant une attention particulière sur les rapports entre institutions et individus (Bilge, 2009; Hankivsky et Christoffersen, 2008). Sur le plan macrosocial, elle met en lumière les manières dont les systèmes de pouvoir sont impliqués dans la (re)production et le maintien des inégalités (Bilge, 2009; Henderson et Tieckmyer, 2009). Sur le plan microsocial, elle permet de cerner les effets des structures d'inégalités sur les parcours individuels des acteurs (Bilge, 2009; Henderson et Tieckmyer, 2009). 


\section{Méthodologie}

Notre recherche porte sur l'expérience de mères autochtones et racisées ayant vécu de la violence conjugale et s'appuie sur une méthodologie qualitative. L'étude s'est déroulée dans deux sites de recherche : dans les Prairies (23 femmes autochtones) et à Montréal (20 femmes racisées). Cependant, compte tenu du propos de cet article eu égard à l'oppression linguistique, nous nous centrerons sur les données recueillies exclusivement à Montréal. Conséquemment, les critères d'inclusion sont :

1. s'identifier comme racisées;

2. avoir vécu de la violence conjugale au cours des deux dernières années;

3. être mère d'au moins un enfant mineur.

Les participantes ont été recrutées via des intervenantes œuvrant auprès d'elles. Les données ont été recueillies au moyen d'entretiens semi-dirigés autour des thèmes suivants : 1 . les conditions de vie à travers lesquelles les participantes performent leur maternité en contexte de violence conjugale; 2. leurs rapports avec les services utilisés; 3. l'influence de leur statut (racisée, réfugiée, immigrante) sur leurs conditions de vie. Les entrevues ont été enregistrées, puis retranscrites et analysées à l'aide du logiciel Nvivo.

\section{Profil des informatrices}

Au moment de l'entrevue, l'âge des informatrices recrutées à Montréal variait entre 27 et 52 ans, la majorité se situant dans la trentaine. Trois des 21 femmes étaient toujours en relation avec le conjoint violent (une célibataire, une conjointe de fait et une mariée); 15 étaient séparées et 3 étaient divorcées. Pour 13 de ces 18 répondantes, la rupture avait eu lieu dans la dernière année (moins de 6 mois $=5$ ), la plus récente séparation avait eu lieu 2 semaines et demie avant l'entrevue. La famille la plus nombreuse comptait 4 enfants. L'âge des enfants variait entre 1 mois et 20 ans. Seize mères détenaient la garde complète des enfants; 1 seule était 
en situation de garde partagée. Une femme n'avait pas indiqué qui détenait la garde et 2 avaient la garde commune avec le père dont elles n'étaient pas séparées.

Deux répondantes occupaient un emploi à temps partiel, 2 travaillaient à temps plein et 17 étaient sans emploi. De ce nombre, 6 n'avaient jamais occupé un emploi. Les domaines d'emploi de celles qui travaillaient ou avaient déjà travaillé étaient variés et s'inscrivaient principalement dans les secteurs secondaire et tertiaire. Les revenus d'emploi constituaient la principale source de revenus pour 2 répondantes, tandis que 13 vivaient des prestations d'assistance sociale et des crédits d'impôt pour enfants. Deux participantes ne bénéficiaient d'aucun revenu alors que 4 d'entre elles n'ont pas indiqué la source de leurs revenus. Aucune répondante ne disposait de plus de $40000 \$$ annuellement; le revenu annuel moyen se situait sous les $10000 \$$ pour 12 d'entre elles, entre 10000 et $20000 \$$ pour 2 , sous les $30000 \$$ pour 4 et au-delà de ce montant pour 1 seule.

Trois femmes revendiquaient le statut de personne réfugiée; 1 était sans statut, 10 avaient obtenu leur résidence permanente et 7 détenaient la citoyenneté canadienne. Sept provenaient de l'Asie du Sud, 9 des Antilles et 4 du continent africain (Afrique du Nord et Afrique de l'Ouest). Une participante rencontrée lors du prétest est née au Canada. Douze d'entre elles sont arrivées au Canada il y a plus de 5 ans. L'immigration la plus récente datait de 7 mois avant l'entrevue. Dix-neuf des 21 répondantes s'associaient à une religion en tant que musulmanes, catholiques, adventistes, anglicanes, pentecôtistes, rastafaris, hindouistes ou sikhes.

\section{Analyse des résultats}

Notre étude a permis de dégager que la réalité des mères qui vivent de la violence conjugale n'est pas homogène : leurs contextes de vie et de violence different et elles y réagissent de diverses façons. Nous avançons donc que l'expérience subjective des mères rencontrées se constitue à l'intersection de multiples axes de différenciation sociale, à la fois discursifs et matériels, et 
que l'articulation de ces axes rend possible une agentivité selon laquelle les participantes sont à même de jouer un rôle dans la (re) production et la déstabilisation des discours, des représentations et des interactions sociales en dépit des contraintes exercées à leur endroit. Plusieurs autres textes (Chbat, Damant et Flynn, 2015; Damant, et collab., soumis) abordent les diverses formes d'oppression, mais le présent article porte sur celle qui est de nature linguistique. Nous proposons donc d'illustrer à travers les récits des participantes comment cette dernière affecte différents plans de leur vie, dont leur vécu en tant que mères.

\section{Oppression linguistique à travers le système d'éducation}

Asha, immigrée de Malaisie et mère monoparentale de 4 enfants qui fréquentent l'école française, peine à aider ces derniers à faire leurs devoirs. Elle reçoit peu de soutien de l'école; elle a l'impression que les professeurs ne veulent pas communiquer avec elle en anglais. Cela l'empêche d'assurer un suivi auprès de ses enfants et l'affecte directement dans son rôle de mère :

"I go into the school all the time $[\ldots]$ But the teachers sometimes, I feel they not help [sic]. [...] I cannot understand you know I cannot help them. That's why I'm try [sic] to changing English school, but it's very hard. [...] because, first, in this country, language is a problem. Second, I cannot communicate with anybody [...] Some people speak English you know. Some white people, they know French, they know English also, they never speak you know. Like they refuse to [...] We need help. They [teachers] can help us. Of course if they know, they can write the English paper. When they go into computer, they can translate right away. Right? " (Asha, originaire de Malaisie) 
"Le manque de ressources pour apprendre le français et ainsi pouvoir communiquer avec les divers services (services de garde, écoles) risque d'avoir des effets pour ces femmes, tant dans leur rôle éducatif auprès de leurs enfants que dans leur intégration avec les diverses ressources éducatives. "
Certaines mères tentent de trouver des outils pour aider leurs enfants à l'école, et ce, malgré le fait qu'elles maitrisent très peu, sinon pas du tout, le français :

«My kids, they are going to French school [...] I use a dictionary to help them from English to French $[\ldots]$ I understand the words, but I can't speak French.» (Vanaja, originaire du Sri Lanka)

Le manque de ressources pour apprendre le français et ainsi pouvoir communiquer avec les divers services (services de garde, écoles) risque d'avoir des effets pour ces femmes, tant dans leur rôle éducatif auprès de leurs enfants que dans leur intégration avec les diverses ressources éducatives. S'ajoutent à cela les réticences de ces mêmes ressources à communiquer dans une langue autre que le français.

\section{Oppression linguistique en lien avec le statut de personne réfugiée}

Plusieurs répondantes étaient en attente d'un statut de personne réfugiée, telle Nana originaire de l'île de Saint-Vincent et mère monoparentale d'un enfant, ce qui complexifie son accès à des cours de français et l'empêche par le fait même de trouver un emploi. Cette participante n'arrive pas à obtenir une place en garderie pour son enfant, les personnes responsables de ces établissements craignant qu'elle ne puisse en payer les frais en raison de son statut jugé précaire. Ne pouvant faire garder l'enfant, cette mère n'arrive pas à se libérer pour suivre des cours de français et ne peut trouver de travail pour subvenir à leurs besoins mutuels :

"If I have to learn French I have to pay for it. If I have to do the French, I have to get someone to watch my daughter; I have to pay for that. Where am I getting all this money from? You know what I mean? [...] Because of my status [refugee status]. What they're trying to say is that 
"...le statut de

personne réfugiée, qui

prend de plus en plus

de temps à obtenir,

limite l'accès aux

ressources tant pour

elles (apprentissage

du français, emploi)

que pour leurs enfants

(services de garde). » they don't know if I'm gonna be able to pay them and they don't know if I have a stable job. But then they also don't understand that with the opportunity of getting my daughter into daycare, I have options. I could find other things to do. [...] At least I could have start there, do my French, and look for proper job in the process." (Nana, originaire de l'île Saint-Vincent)

Ainsi, pour plusieurs participantes, le statut de personne réfugiée, qui prend de plus en plus de temps à obtenir, limite l'accès aux ressources tant pour elles (apprentissage du français, emploi) que pour leurs enfants (services de garde).

\section{Oppression linguistique en lien avec les services reçus}

"Faute de parler le français ou de le maîtriser quelque peu, certaines participantes ont reconnu avoir reçu des services inadéquats pour leurs besoins, notamment en garderie, à l'école et auprès des agences gouvernementales d'aide financière. "
Faute de parler le français ou de le maîtriser quelque peu, certaines participantes ont reconnu avoir reçu des services inadéquats pour leurs besoins, notamment en garderie, à l'école et auprès des agences gouvernementales d'aide financière. Ces obstacles peuvent avoir des répercussions directes sur leur bien-être. C'est le cas de Yellow Flower, une mère monoparentale originaire des Bahamas qui n'a pas réussi à recevoir ses prestations d'assurances sociales au moment souhaité parce que l'agente qui gérait son dossier ne parlait pas anglais :

"I know a little bit of French, but it's not enough to hold a real conversation [...] I tried to get welfare but they giving me a very hard time again, because the person speaks French, but very, barely any English, you know. And I'm wondering why... Why is a government person in a government office not willing to speak English to me [...] It's the welfare. It's the government. " (Yellow Flower, originaire des Bahamas) 
"Ainsi, un statut similaire sur l'axe de différenciation sociale qu'est la " race » ne garantit pas une ouverture ou la nonreproduction d'autres formes d'oppression sur d'autres axes, tels que celui de la langue..."
Notons que la résistance à offrir des services gouvernementaux en anglais est perpétuée dans ce cas-ci par une employée d'origine haïtienne qui partage elle-même une expérience de racialisation dans le contexte majoritairement blanc du Québec. Ainsi, un statut similaire sur l'axe de différenciation sociale qu'est la " race " ne garantit pas une ouverture ou la non-reproduction d'autres formes d'oppression sur d'autres axes, tels que celui de la langue :

"You know. I'm not gonna say that it's because of my race, because I did have another woman that this is the second person I got. The first one I got was I'm sure she was from Haiti $[. .$.$] So$ she's a woman of minority too, you know [...] I mean, we oppress each other too sometimes.» (Yellow Flower, originaire des Bahamas)

\section{La « race » une affaire de langue}

"L'oppression

linguistique vécue par ces femmes peut être perçue comme une forme de racisme linguistique..."
L'oppression linguistique vécue par ces femmes peut être perçue comme une forme de racisme linguistique (McCall, 1992). Souvent motivée par des représentations réductrices et homogénéisantes à l'endroit des nouveaux arrivants qui ne parlent pas le français, l'oppression linguistique est manifeste dans les interactions de plusieurs répondantes avec des acteurs provenant de milieux variés. Les témoignages suivants soulèvent bien le caractère raciste de l'oppression linguistique qu'elles ont vécue du fait qu'elles ne parlaient pas la langue d'établissement du Québec :

"At a point in time I was thinking not with the colour of my skin, I was thinking that I am being treated like this because I don't speak French. Because in some places you go and you don't speak French, you don't have that much respect and people don't really wanna speak to you. As same as all you crave to learn my language, I crave to learn your language, too.You know, and 
I'm not dummy. [...] I speak English, you speak French. English and French, none is better than none.Your French is not better than my English. No. " (Cam, originaire de l'île Saint-Vincent)

"Sometimes [...] I try not to bring race into anything, or ethnicity into anything, but it's obvious that sometimes those things do exist, because a lot of time people think that oh you come from the Caribbean [...] or you file refugee claim and all you wanna do is sit down and live off the system, you know what I mean. And so there's a bias sometimes towards women of minority, like ethnic, you know, different ethnic background and immigrants [...] The lady [assistances sociales] just keeps saying to me: "No, you, no money for you." Like she wouldn't speak English properly, and she can very hardly explain to me what she's trying to say. And it was very frustrating.» (Yellow Flower, originaire des Bahamas)

"Les mauvais services qui semblent souvent accompagnés de représentations racistes et dévalorisantes aux yeux des femmes racisées qui ne parlent pas le français peuvent pousser ces dernières à retourner auprès $d u$ conjoint violent, les condamner à un état de précarité extrême ou les empêcher de travailler..."
Les mauvais services qui semblent souvent accompagnés de représentations racistes et dévalorisantes aux yeux des femmes racisées qui ne parlent pas le français peuvent pousser ces dernières à retourner auprès du conjoint violent, les condamner à un état de précarité extrême ou les empêcher de travailler :

«A black woman living in a shelter and you're calling for welfare, they just assume that wow, you're just a bum or something. And you don't even speak French and you lived in Canada. [...] You lived here for 5 years, and you don't speak French. Shame on you. You know [...] it's oppressive, because there's nobody giving you a chance. It makes a woman frustrated and [...] a lot of time it cause people to give up. And revert back to their old thing, that's why a lot of women go back to their partner. You know, 
and stay in the abusive relationship, because it's better to give your child food. You know how hard it is to see your child hungry, cold, because they don't have warm clothes to wear when they standing on the street and you can't provide that, but you know a man is gonna beat me up but at least my child will be warm. » (Yellow Flower, originaire des Bahamas)

\section{Discussion}

"L'intersection de ces formes d'oppression augmente la difficulté des femmes racisées à s'intégrer à la société québécoise, accroit leur dépendance envers leur conjoint et menace leur sécurité et celle de leurs enfants. "
Le présent article porte sur l'oppression linguistique. L'analyse des résultats permet toutefois de voir à quel point cette forme d'oppression interagit avec d'autres, telles que le statut d'immigration, le genre ou la classe sociale. L'intersection de ces formes d'oppression augmente la difficulté des femmes racisées à s'intégrer à la société québécoise, accroit leur dépendance envers leur conjoint et menace leur sécurité et celle de leurs enfants.

En ce qui concerne l'oppression linguistique, les propos de nos participantes reprennent les résultats des quelques études recensées. En effet, à l'instar de Bélanger, Sabourin et Lachapelle (2011), les femmes rencontrées n'avaient pas eu dans leur pays d'origine un contact avec le français et pensaient immigrer dans un pays de langue anglaise. De plus, les ressources en temps et en argent auxquelles elles ont eu droit étaient limitées et ont nui à leur intégration, notamment avec les milieux scolaires, les lieux de travail et les services d'aide financière.

Tout comme Piché (1992), Hohl (1996) et Mc Andrew et Jacquet (1996), nous avons entendu les propos des femmes sur le personnel enseignant des écoles de leurs enfants et sur les employés des services d'aide sociale qui exigent qu'elles partagent les mêmes normes sociales et culturelles en ce qui a trait à la participation au projet éducatif des enfants et au vivre-ensemble. Ainsi, nous soutenons à l'instar de Bataille, Mc Andrew et Potvin (1998, p. 130), et ce, seize ans plus tard, que l'« inadaptation des politiques scolaires et des contenus d'enseignement ", l'« incompréhension 
"...elles se retrouvent pour la plupart dans une situation où leur non-connaissance de la langue française - dans un contexte où l'intégration des personnes racisées, réfugiées et de culture non chrétienne est de plus en plus difficile les conduit dans une dépendance à l'égard de leurs conjoints tant sur le plan économique que social, ce qui ne fait que les isoler davantage et les maintenir dans une situation de violence conjugale. » culturelle " et les «tensions interethniques » sont en cause. Il n'est donc pas étonnant que nos répondantes éprouvent du ressentiment envers la société d'établissement et qu'elles sentent qu'elles n'ont pas reçu l'aide dont elles avaient besoin, à la fois pour s'intégrer à la société québécoise et pour sortir de la violence dont elles étaient victimes.

Nous reconnaissons également dans les entrevues, des propos qui s'apparentent à ce que McCall (1992) nomme la "racialisation de la langue ». N'ayant pas une bonne connaissance du français, ces femmes se retrouvent à la fois dans une situation de précarité économique et de dépendance à l'égard du conjoint violent. Ces situations sont encore plus difficiles à vivre lorsqu'elles n'ont pas encore obtenu leur statut de personne réfugiée.

Ainsi, elles se retrouvent pour la plupart dans une situation où leur non-connaissance de la langue française - dans un contexte où l'intégration des personnes racisées, réfugiées et de culture non chrétienne est de plus en plus difficile - les conduit dans une dépendance à l'égard de leurs conjoints tant sur le plan économique que social, ce qui ne fait que les isoler davantage et les maintenir dans une situation de violence conjugale.

\section{Conclusion}

Nous avançons que la perspective féministe intersectionnelle est particulièrement pertinente pour soulever et analyser des enjeux liés à l'oppression linguistique que rencontrent les femmes racisées en contexte québécois. Peu de recherches empiriques ont été faites jusqu'à présent en utilisant cette perspective théorique et du travail reste à faire pour affiner les analyses. Cela dit, notre analyse nous permet de conclure qu'aussi diverses soient-elles, les expériences de violence vécues par les femmes se réalisent au sein de contextes sociohistoriques sur lesquels il est impératif de s'attarder. Bien que les femmes racisées rencontrées aient des expériences qui sont propres à chacune, ces dernières convergent sous certains aspects, dont celui de l'intégration linguistique. Il est 
difficile de décrire à partir de leurs témoignages l'interaction entre les divers domaines de pouvoir, qu'ils soient d'ordre structurel, organisationnel, hégémonique ou interpersonnel (Collins, 2000) et les impacts de chacun d'eux. Cependant, leur discours nous permet de voir l'interaction entre l'oppression linguistique, le statut de personne réfugiée et la classe sociale, et comment cette interaction maintient ces femmes dans un isolement et une dépendance à l'égard du conjoint violent. Pour cette raison, nous pensons qu'il est important de formuler les recommandations suivantes :

- Il faut tout d'abord souligner la nécessité de bien former les personnes qui auront à intervenir auprès de communautés linguistiques et ayant une appartenance minoritaire au Québec (enseignants, travailleurs sociaux, policiers, agents de sécurité du revenu, intervenantes en maison d'hébergement, etc.). Ces formations doivent viser à dénoncer toutes manifestations préjugées en fonction de la langue, de la race et plus récemment à l'égard de la religion, plus particulièrement de l'islam.

- Un soutien financier offert aux personnes immigrantes ne maitrisant pas encore le français leur faciliterait l'apprentissage de la langue. Des initiatives importantes visant à éviter le racisme linguistique doivent aussi être entreprises. Dans le cas des femmes victimes de violence conjugale, les services d'interprète, d'apprentissage du français et de soutien financier, tout particulier quand les femmes sont dépendantes de leurs conjoints, doivent être explicités, maintenus et augmentés.

- Il ne faut surtout pas ignorer les rapports d'inclusion et d'exclusion entre les communautés linguistiques et les groupes ayant un statut minoritaire au Québec, surtout dans une période où les projets néolibéraux pourraient dresser les communautés les unes contre les autres en période de rareté budgétaire.

\section{Notes}

Mothering in the context of domestic violence in Canada and the United Kingdom: A focus on Aboriginal and Black Minority Ethnic women (Damant, et collab., CRSH, 2009-2012). 
2 Un autre article a été consacré à la mise en lumière des multiples formes de l'oppression subie par nos répondantes (Chbat, Damant et Flynn, 2015). L'équipe de recherche travaille actuellement à la rédaction d'un troisième article dans lequel les différentes stratégies de résistance qui ont été mobilisées par ces femmes ont été mises de l'avant pour contrer les multiples rapports de domination qu'elles ont rencontrés. Le présent article soulève d'ailleurs certains exemples de stratégies de résistance en lien avec la langue majoritaire au Québec. Le troisième article souhaite aborder ces stratégies de façon plus large.

\section{Notes biographiques}

- Dominique Damant, Ph. D. est professeure titulaire à l'École de service social de l'Université de Montréal. Elle travaille sur la violence faite aux femmes analysée avec le féminisme intersectionnel.

- Jeanne Bernard est étudiante à la maitrise à l'École de service social de l'Université de Montréal. Son mémoire porte sur l'implication des étudiantes en travail social au printemps érable.

- Marianne Chbat est étudiante au doctorat en sciences humaines appliquées à l'Université de Montréal. Ses intérêts sont liés aux questions de genre, de sexualités, d'ethnicité et de violence, dans une perspective intersectionnelle.

- Catherine Flynn Ph.D. vient de compléter son doctorat à l'École de service social de l'Université de Montréal. Sa thèse porte sur une analyse intersectionnelle de l'expérience de la violence structurelle vécue par des jeunes femmes marginalisées.

\section{Bibliographie}

BATAILLE, Philippe, Marie MCANDREW et Maryse POTVIN (1998). «Racisme et antiracisme au Québec : analyse et approches nouvelles ", Cahiers de recherche sociologique, Vol.3, № 1, p. 115-144.

BÉLANGER, Alain, Patrick SABOURIN et Réjean LACHAPELLE (2011). "Une analyse des déterminants de la mobilité linguistique intergénérationnelle des immigrants », Cahiers québécois de démographie, Vol. 40, № 1, p. 113-138.

BILGE, Sirma (2009). « Les théorisations féministes de l'intersectionnalité », Diogène, Vol. 1, № 225, p. $70-88$.

BRAH, Avtar, et Ann PHEONIX (2004). "Ain't I a woman: Revisiting intersectionality ", Journal of International Women's Studies, Vol. 5, No 3, p. 75-86.

BRÉDIMAS-ASSIMOPOULOS, Nadia (1983). «Dynamique ethnique et évolution socio-politique du Québec : le cas de la population grecque », Sociologie et sociétés, Vol. 15 N² 2, p. 105-116.

CALINON, Anne-Sophie (2009). Facteurs linguistiques et sociolinguistiques de l'intégration en milieu multilingue : le cas des immigrants à Montréal, [thèse de doctorat], Montréal, Canada, Université de Montréal, $357 \mathrm{p}$.

CASTONGUAY, Charles (1997). «Évolution de l'assimilation linguistique au Québec et au Canada entre 1971 et 1991 ", Recherches sociographiques,Vol. 38, № 3, p. 469-490. 
CHBAT, Marianne, Dominique DAMANT et Catherine FLYNN (2015). «Analyse intersectionnelle de l'oppression des mères racisées en contexte de violence conjugale : mise en application de la matrice de pouvoir ", Nouvelles pratiques sociales, Vol. 6, $\mathrm{N}^{\circ}$ 2, p. 97-110.

COLLINS, Patricia Hill (2000 [1990]). Black feminist thought: Knowledge, consciousness, and the politics of empowerment, New York, Routledge, 335 p.

COLLINS, Patricia Hill (1998). Fighting words: Black women and the search for justice, Minneapolis, University of Minnesota Press, $312 \mathrm{p}$.

CRENSHAW, Kimberlé (1989). «Demarginalizing the intersection of race and sex:A Black feminist critique of discrimination doctrine ", Feminist Theory and Antiracist Practice, University of Chicago Legal Forum, No 89, p. 138-167.

DAMANT, Damant, et collab. (soumis). «Money counts:A feminist intersectional analysis of racialized and First Nation mothers living in the context of domestic violence ", British Journal of Social Work.

DAMANT, Dominique, et Simon LAPIERRE (2012). "Introduction ", dans Dominique Damant et Simon Lapierre (dirs.), Regards critiques sur la maternité dans divers contextes sociaux, Québec, Presse de l'Université du Québec, p. 1-5.

DAMANT, Damant, et collab. (2008). «Taking child abuse and mothering into account: Intersectional feminism as an alternative for the study of domestic violence ", Affilia,Vol. 23, No 2, p. 123-185.

DOBASH, Rebecca Emmerson, et Russell P.DOBASH (1979).Violence against wives:A case against the patriarchy, New York, The Free Press, 339 p.

DOBASH, Rebecca Emmerson, et Russell P. DOBASH (2000). «The politics and policies of responding to violence against women ", dans Jalna Hanmer et Catherine Itzin (dires.), Home Truths About Domestic Violence, Londres, Routledge, p. 187-204.

FORTIN, Sylvie (2001). "Citoyennetés et appartenances en situation de migration ", Les Cahiers du Gres,Vol. 2, No 1, p. 73-84.

GAGNÉ, Caroline (2010). La construction discursive de l'identité en contexte migratoire. Une étude de cas : les "enfants de la Loi 101 ", [mémoire de maîtrise], Québec, Canada, Université Laval, 131 p.

HANKIVSKY, Olena, et Ashlee CHRISTOFFERSEN (2008). "Intersectionality and the determinants of health: a Canadian perspective ", Critical Public Health,Vol. 18, № 3, p. 271-283.

HENDERSON, Debra, et Ann TICKAMYER (2009). "The intersection of poverty discourses: Race, class, culture and gender ", dans Bonnie Thornton Dill et Ruth Enig Zambana (dires.), Emerging intersections: Race, class and gender in theory, policy and practice, New Brunswick, Rutgers, p. 73-100.

HELLY, Denise (1994). "Politiques à l'égard des minorités immigrées », Sociologie et sociétés, Vol. 26, No 2, p. 127-144.

HOHL, Janine (1996). "Qui sont "les parents"? Le rapport de parents immigrants analphabètes à l'école ", Lien social et Politiques, № 35, p. 51-62.

HUMPHREYS, Cathy, et collab. (2006). «Talking to my mum: Developing Ccommunication between women andc in the aftermath of domestic violence ", Journal of Social Work,Vol. 6, № 1, p. 53-65.

IMMIGRATION, DIVERSITÉ ET INCLUSION QUÉBEC (2014). Apprendre le français au Québec, réf. du 21 novembre 2014, http://www.immigration-quebec.gouv.qc.ca/fr/langue-francaise/apprendre-quebec

KANOUTÉ, Fasal, et collab. (2008). «Familles et réussite scolaire d'élèves immigrants du secondaire », Revue des sciences de l'éducation, Vol. 34, No 2, p. 265-289. 
KRANE, Julia, et Linda DAVIES (2007). « Mothering under difficult circumstances: Challenges to working with battered women ", Affilia,Vol. 22, No 1, p. 23-38.

LABELLE, Micheline (1989). «Immigration, main-d'oeuvre immigrée et société d'accueil : réalités et contradictions ", Nouvelles pratiques sociales, Vol. 2, № 2, p. 107-122.

LABELLE, Micheline (1990). "Immigration, culture et question nationale ", Cahiers de recherche sociologique, $\mathrm{N}^{\circ} 14$, p. 143-151.

MC ANDREW, Marie, et Marianne JACQUET (1996). « Le discours public des acteurs du monde de l'éducation sur l'immigration et l'intégration des élèves des minorités ethniques ", Recherches sociographiques, Vol. 37, № 2, p. 279-299.

MCALL, Christopher (1992). «Langues et silence : les travailleurs immigrés au Québec et la sociologie du langage ", Sociologie et sociétés, Vol. 24, № 2, p. 117-130.

MOLINARO, Ines, et Catherine EGO (1999). «Contexte et intégration. Les communautés allophones au Québec ", Globe : revue internationale d'études québécoises, Vol. 2, № 2, p. 101-124.

OFFICE QUÉBÉCOIS DE LA LANGUE FRANÇAISE (2013). Chronologie de la législation linguistique au Québec, réf. du 21 novembre 2014, http://www.oqlf.gouv.qc.ca/charte/reperes/reperes.html

OFFICE QUÉBÉCOIS DE LA LANGUE FRANÇAISE (2014). Charte de la langue française, réf. du 21 novembre 2014, http://www2.publicationsduquebec.gouv.qc.ca/dynamicSearch/telecharge. php?type $=2$ etfile $=/$ C_11/C11.html

OUELLET, Rémi (2011). Comportement linguistique des immigrants allophones et caractéristiques, [mémoire de maitrise], Montréal, Canada, Université de Montréal. 80 p.

PEDERSEN, Jeanette Somlak, Lorraine Halinka MALCOE et Jane PULKINGHAM (2013). «Explaining Aboriginal/Non-Aboriginal inequalities in postseparation violence against Canadian women: application of a structural violence approach ", Violence against women, Vol. 19, № 8, p. 1034-1058.

PICHÉ,Victor (1992). «Débat — Le discours démo-politique au Québec :inclusion ou exclusion? », Sociologie et sociétés, Vol. 124, № 2, p. 143-150.

PRESNUKHINA, Yulia (2011). Intégration linguistique des immigrants au marché du travail au Québec, [mémoire de maitrise], Montréal, Canada, Université de Montréal, 80 p.

PRINS, Baukje (2006). "Narrative accounts of origins: A blind spot in the intersectional approach ", European Journal of Women's Studies, Vol. 13, № 3, p. 277-290.

REICHHOLD, Stephan, et André JACOB (2012). "Intégration des immigrants : un problème de ressources ou de finalités? ", Relations,Vol. 757, p. 36-37.

STASIULIS, Daiva (1999). «Feminist intersectional theorizing ", dans Peter Li (dir.), Race and ethnic relations in Canada, Toronto, Oxford UP, p. 347-398.

TURCOTTE, Yvan (1997). "L'immigration et l'intégration des immigrants au Québec au cours des quinze dernières ", Nouvelles pratiques sociales, Vol. 10, No 1, p. 53-57. 